ISSN 0103-5150

Fisioter. Mov., Curitiba, v. 23, n. 3, p. 461-471, jul./set. 2010

Licenciado sob uma Licença Creative Commons

\title{
Qualidade de vida: pacientes com insuficiência renal crônica no município de Caruaru, PE
}

Life quality: patients with chronic renal failure in the city of Caruaru, PE

\author{
Patrícia Bezerra da Costa $^{[a]}$, Karla Fabiana da Silva Vasconcelos ${ }^{[b]}$, Rafael Miranda Tassitano ${ }^{[\mathrm{c}]}$
}

[a] Graduada em Fisioterapia pela Associação Caruaruense de Ensino Superior (ASCES), Caruaru, PE - Brasil, e-mail: patybz202@hotmail.com

[b] Graduada em Fisioterapia pela Associação Caruaruense de Ensino Superior (ASCES), Pós-Graduanda em Saúde Pública pela Universidade de Pernambuco (UFPE), Caruaru, PE - Brasil, e-mail: kfsv_pe@hotmail.com

[c] Mestre, Doutorando em Nutrição pela Universidade Federal de Pernambuco (UFPE), Recife, PE - Brasil, e-mail: rafael.tassitano@gmail.com

\section{Resumo}

Introdução: Qualidade de vida é uma dimensão que tem sido amplamente investigada na saúde da população, independente da faixa etária. Uma condição crônica é um fator que interfere na realização das atividades da vida diária e na percepção de bem-estar individual, como é o caso dos pacientes em tratamento por hemodiálise. Objetivo: Descrever as características e analisar as possíveis associações entre a Insuficiência Renal Crônica (IRC) e a qualidade de vida em uma amostra representativa de pacientes com o agravo no município de Caruaru, PE. Método: A pesquisa foi realizada nos centros de tratamentos dialíticos na cidade de Caruaru, onde toda a população com IRC é atendida ( $\mathrm{n}=192)$, sendo 82 pacientes do Centro Regional de Hemodiálise do Agreste (CRHA) e 110 da clínica SOS Rim - Clínica Nefrológica de Caruaru. Para a composição da amostra foi realizado um censo e todos os pacientes cadastrados foram pré-selecionados inicialmente para o estudo. Resultados: Verificou-se que a maioria dos participantes relatou algum grau de incômodo: dores musculares $(69,4 \%)$, cãibras $(89,8 \%)$, coceiras $(67,3 \%)$, fraqueza muscular $(73,5 \%)$, diminuição de líquido $(91,8 \%)$, diminuição alimentar $(85,7 \%)$ e capacidade de trabalhar $(75,5 \%)$. Já quando questionados se o esforço físico interfere na sua qualidade de vida, este estudo revelou que 70,3\% afirmam que sim. Em relação à saúde, 80,6\% das pessoas dizem que a doença renal crônica interfere de forma negativa na qualidade de vida. Conclusão: Os dados indicam que pacientes com IRC apresentam diminuição na qualidade de vida graças a repercussões musculoesqueléticas, tais como cãibras, fraqueza muscular e esforço físico.

Palavras-chave: Qualidade de vida. Insuficiência renal crônica. KDQOL-SF. 


\begin{abstract}
Introduction: Life quality has been a subject of growing research in studies of population health, in all times of ages. A chronic condition is a factor that interferes with the conduct of activities of daily living and the perception of individual well-being, as is the case of patients being treated by hemodialysis. Objective: To describe the characteristics and to analyze the possible associations between the CRF and life quality related to health in a representative sample of patients which was worsted in the city of Caruaru, PE. Method: The survey was conducted in dialysis treatment centers in Caruaru city, where all population with CRF is attended ( $n=192)$, being 82 patients of the Regional Hemodialysis Center (Centro Regional de Hemodiálise do Agreste - CRHA) and 110 patients of the SOS Rim Clinic-Caruaru's Nephrological Clinical. For the composition of the sample was accomplished a census and all patients enrolled were initially preselected first for study. Results: It was verified that the majority of study participants reported some degree of discomfort: muscle pain $(69.4 \%)$, cramps $(89.8 \%)$, itching $(67.3 \%)$, weakness $(73.5 \%)$, reduced net $(91.8 \%)$, decreased food $(85.7 \%)$ and ability to work $(75.5 \%)$. When asked if the physical effort interferes with their quality of life, this study revealed that $70.3 \%$ say so. In relation to health, $80.6 \%$ of people say that kidney disease interferes in a negative way on the quality of life. Conclusion: The data in this study indicate that patients with CRF have decreased in life quality due to musculoskeletal repercussions such as cramps, muscle weakness and physical effort.
\end{abstract}

Keywords: Life quality. Chronic renal failure. KDQOL-SF.

\title{
Introdução
}

A investigação sobre a qualidade de vida (QV) é um tema de investigação crescente em estudos de saúde (1). O estudo sobre QV vem assumindo importância sob vários aspectos, nos últimos anos, particularmente no que diz respeito à sua avaliação e mensuração, quer individualmente, quer coletivamente.

A avaliação da qualidade de vida relacionada à saúde (QVRS) tem sido uma prática cada vez mais frequente na medicina atual. Esta avaliação objetiva monitorar a saúde de uma determinada população, diagnosticar a natureza, gravidade e prognóstico da doença, além de avaliar os efeitos do tratamento (2).

Recentemente, a avaliação da QVRS dos portadores de doenças crônicas tem sido alvo de grande atenção, pois a percepção de melhora ou piora dos doentes crônicos pode auxiliar no tratamento da doença. Existe uma preocupação em identificar o quanto a condição crônica interfere na realização das atividades da vida diária e na percepção de bem-estar individual (3), como é o caso dos pacientes em tratamento por hemodiálise (4). No início do comprometimento da função renal, o indivíduo apresenta-se assintomático. A insuficiência renal torna-se crônica quando há deterioração irreversível da função renal e elevação persistente da creatinina no organismo. Isso ocorre por falha na capacidade do organismo em manter o equilíbrio metabólico e eletrolítico, ocasionando a uremia (5).

Atualmente, a doença renal crônica (DRC) é considerada um problema mundial de saúde pública (6-10). A insuficiência renal crônica (N18 e N19 da CID-10) é a perda lenta e irreversível das funções renais(11), portanto compreende desde efeitos leves controlados à base de medicamento e dieta, até o estágio no qual o rim chega a ter sua função normal reduzida em mais de $90 \%$, quando então se indica a diálise ou o transplante renal. A ausência de tais intervenções leva o paciente ao óbito em 72 horas (12). Essa doença resulta em inúmeros processos adaptativos que em sua maioria afetam diretamente a qualidade de vida do paciente, sendo que este sofre várias restrições decorrentes da doença e do tratamento (13).

Os indivíduos em tratamento hemodialítico apresentam melhores resultados no domínio psicológico, por acreditarem que o transplante renal seja a cura para a doença (14). O transplante renal geralmente está associado a uma diminuição na prevalência de transtornos mentais (depressão). Contudo, essa diminuição pode demorar alguns meses para se efetivar, podendo haver aumento da incidência no início, demonstrando uma dificuldade inicial no processo de adaptação à nova situação (15). 
A hemodiálise (HD), como um tratamento para insuficiência renal crônica (IRC), substitui as principais funções do rim, mas não é capaz de substituir integralmente o órgão (16). Ela se constitui em um procedimento de alto custo/complexidade que envolve uma assistência altamente especializada, tecnologia avançada, ações de alta complexidade e requer articulação entre os níveis secundário e terciário da assistência. Além disso, apresenta, ao longo dos últimos anos, uma demanda crescente, o que tem implicado considerável consumo de recursos financeiros (17).

No Brasil, existem 41.614 pacientes em terapia renal substitutiva, conforme o Censo da Sociedade Brasileira de Nefrologia de 2006, distribuídos por regiões Sudeste 57,4\% $(\mathrm{N}=23.880)$, Nordeste 19,1\% $(\mathrm{N}=$ 7.948), Sul 15,4\% ( $=6.394)$, Centro-oeste 5,8\% $(\mathrm{N}=2.420)$ e Norte $2,3 \%(\mathrm{~N}=972)$ sendo $35.928(89,4 \%)$ pacientes em hemodiálise e 3.963 (10,6 \%) pacientes em outros tipos de diálise (18). A Sociedade Brasileira de Nefrologia (SBN) estima que a cada ano somente 2.700 brasileiros são submetidos ao transplante renal. Os pacientes transplantados têm maior possibilidade de realizar um trabalho remunerado, visto que não interrompem suas atividades cotidianas para dialisar (12).

Além da sobrevida dos pacientes em diálise, alguns outros indicadores (tais como taxas de incidência e prevalência de pacientes em diálise e outros índices de morbidade) são utilizados para avaliar fatores demográficos e clínicos dos pacientes, bem como as características das intervenções terapêuticas. Entretanto, sabe-se que também é importante a abordagem de outros aspectos relevantes para a vida desses pacientes, tais como a reabilitação funcional, psicológica, socioeconômica e familiar (19). Cabe aos profissionais de saúde promover medidas que possam minimizar as alterações na saúde do renal crônico submetido à hemodiálise (20).

O Kidney Disease and Quality-of-Life Short-Form (KDQOL-SF) é um instrumento específico que avalia doença crônica, aplicável a pacientes que realizam algum tipo de programa dialítico. É um instrumento auto-aplicável de 80 itens, divididos em 19 escalas.

Desta forma, avaliações periódicas dos pacientes poderão ser feitas, e aqueles com piores escores de qualidade de vida poderão ser identificados e eventualmente receber suporte especializado. O questionário poderá ainda ser útil para avaliar pacientes que serão submetidos ao transplante renal (21).

O interesse para o desenvolvimento deste estudo advém da lacuna no conhecimento e escassez de levantamento de dados regionais que pudessem fornecer informações sobre a qualidade de vida do paciente renal crônico. Sendo assim, este estudo teve como objetivo descrever as características e analisar as possíveis associações entre a IRC e a qualidade de vida relacionada à saúde em uma amostra representativa de pacientes do município de Caruaru, PE.

\section{Materiais e métodos}

\section{Caracterização do estudo}

O delineamento adotado foi de um estudo epidemiológico, descritivo, do tipo transversal.

\section{População e composição da amostra}

A pesquisa foi realizada nos centros de tratamentos dialíticos no município de Caruaru, PE, onde toda a população com IRC é atendida ( $\mathrm{n}=192$ ), sendo 82 pacientes do Centro Regional de Hemodiálise do Agreste (CRHA), mantido pelo Estado, e 110 da clínica particular SOS Rim - Clínica Nefrológica de Caruaru. Para a composição da amostra foi realizado um censo e todos os pacientes cadastrados foram pré-selecionados inicialmente para o estudo.

Como critérios de inclusão foram considerados:

a) pacientes com mais de seis meses de tratamento; 
b) pacientes com capacidade de responder o questionário;

c) pacientes com parâmetros hemodinâmicos estáveis;

d) pacientes que não apresentavam nenhum tipo de deficiência física ou mental.

Assim, participaram do estudo 49 pacientes, sendo 32 do CRHA e 17 do SOS Rim. Observou-se que 93 indivíduos não tinham o tempo mínimo de seis meses de diálise; 14 eram analfabetos; 5 com deficiências (3 físicas, 1 visual e 1 auditiva); 4 hemodinamicamente instáveis; 1 transplantado; 4 transferidos; 5 óbitos; e 17 se recusaram a participar.

\section{Implementação do estudo}

O trabalho foi aprovado pelo Comitê de Ética em Pesquisa (CEP) da Associação Caruaruense de Ensino Superior (ASCES), protocolo n. 012/08. Os pacientes foram informados sobre as características do estudo e aceitaram participar voluntariamente - conforme determina a Resolução n. 196/96 do Conselho Nacional de Saúde, sobre a pesquisa envolvendo seres humanos - dos protocolos de coleta de dados (questionários) do estudo. Foram atendidas as recomendações sugeridas pelos componentes da banca examinadora, e uma carta foi enviada aos diretores responsáveis pelas instituições selecionadas para participar do estudo, a fim de obter apoio e autorização necessários à realização da coleta de dados.

\section{Procedimento}

As entrevistas foram feitas aos pacientes durante a hemodiálise de forma individual, com duração média de 20 minutos. Os pacientes foram selecionados por meio de uma avaliação dos prontuários e posteriormente foram aplicados os questionários para identificação do nível socioeconômico (ABEP, 1997) e para avaliação da qualidade de vida em pacientes renais crônicos, o Kidney Disease and Quality-of-Life Short-Form (KDQOL- SF). Validado e adaptado no Brasil por Duarte (22), este é provavelmente o questionário mais completo disponível atualmente para avaliar qualidade de vida de pacientes com insuficiência renal crônica, pois inclui aspectos genéricos e específicos relativos à doença renal.

\section{Procedimentos de crítica e tabulação de dados}

O procedimento de tabulação final dos dados foi efetuado através do programa Epi Data, um sistema de domínio público disponível em www.epidata.dk.

\section{Análise dos dados}

Na sequência à tabulação e conferência dos dados, as informações foram transportadas e analisadas com auxílio de programa estatístico SPSS ${ }^{\circledR}$ versão 11.0. Inicialmente, foram utilizados os recursos da estatística descritiva: média, desvio padrão, frequência relativa e intervalo de confiança 95\%. Para o estudo dos determinantes associados aos níveis de QV, foi realizada uma categorização das variáveis percepção e saúde com doença renal interfere demais na vida e gasto de tempo com a doença; gasto de tempo com a doença com esforço físico e idade, transformando-as em binárias (positivo e negativo). Recorreu-se inicialmente à análise de Qui-quadrado para associação; quando pertinente, utilizou-se também o teste de Fisher para amostras pequenas. Foi considerado um $\mathrm{p}<0,05$ para nível de significância. 


\section{Resultados}

A amostra final foi composta por 49 pacientes com a idade variando entre 20 e 88 anos, sendo o maior número de pacientes $(46,9 \%)$ com idades superiores a 50 anos. Um pouco mais da metade da amostra foi composta por mulheres (57,1\%), como constata o trabalho de Pacheco (23).

Em relação ao grau de incômodo verificou-se que 69,4\% sentem dores musculares, cãibras (89,8\%), coceiras (67,3\%), fraqueza muscular $(73,5 \%)$, diminuição da ingestão de líquido $(91,8 \%)$, diminuição da ingestão alimentar $(85,7 \%)$ e capacidade de trabalhar $(75,5 \%)$, conforme apresentado na Tabela 1.

Tabela 1 - Relação entre incômodo e doença renal

\begin{tabular}{|c|c|c|c|c|c|c|c|c|c|c|}
\hline & \multicolumn{2}{|c|}{ Não incomoda } & \multicolumn{2}{|c|}{$\begin{array}{l}\text { Incomoda } \\
\text { Pouco }\end{array}$} & \multicolumn{2}{|c|}{$\begin{array}{c}\text { Incomoda } \\
\text { Moderadamente }\end{array}$} & \multicolumn{2}{|c|}{$\begin{array}{l}\text { Incomoda } \\
\text { Muito }\end{array}$} & \multicolumn{2}{|c|}{$\begin{array}{c}\text { Incomoda } \\
\text { Extremamente }\end{array}$} \\
\hline & $\mathrm{n}$ & $f(\%)$ & $\mathbf{n}$ & $f(\%)$ & n & $\mathrm{f}(\%)$ & $\mathbf{n}$ & $f(\%)$ & $\mathrm{n}$ & $f(\%)$ \\
\hline Dores musculares & 15 & 30,6 & 17 & 34,7 & 7 & 14,3 & 7 & 14,3 & 3 & 6,1 \\
\hline Dor no peito & 30 & 61,2 & 10 & 20,4 & 4 & 8,2 & 4 & 8,2 & 5 & 10,2 \\
\hline Cãibras & 5 & 10,2 & 7 & 14,3 & 6 & 12,2 & 17 & 34,7 & 14 & 28,6 \\
\hline Coceira & 16 & 32,7 & 11 & 2,4 & 5 & 10,2 & 15 & 30,6 & 2 & 4,1 \\
\hline Pele seca & 18 & 36,7 & 10 & 20,8 & 6 & 12,2 & 14 & 28,6 & 1 & 2,0 \\
\hline Falta de ar & 26 & 53,1 & 4 & 8,2 & 2 & 4,1 & 12 & 24,5 & 5 & 10,2 \\
\hline Fraqueza & 13 & 26,5 & 10 & 20,4 & 5 & 10,2 & 17 & 34,7 & 3 & 6,1 \\
\hline Falta apetite & 21 & 42,9 & 8 & 16,3 & 1 & 2,0 & 13 & 26,5 & 2 & 4,1 \\
\hline Muito cansado & 14 & 28,6 & 13 & 26,5 & 3 & 6,1 & 15 & 30,6 & 4 & 8,2 \\
\hline Dormência & 24 & 49,0 & 4 & 8,2 & 2 & 4,1 & 15 & 30,6 & 3 & 6,1 \\
\hline Vontade de vomitar & 17 & 34,7 & 4 & 8,2 & 1 & 2,0 & 18 & 36,7 & 9 & 18,4 \\
\hline $\begin{array}{l}\text { Diminuição de } \\
\text { líquido }\end{array}$ & 4 & 8,2 & 8 & 16,3 & 4 & 8,2 & 12 & 24,5 & 21 & 42,9 \\
\hline $\begin{array}{l}\text { Diminuição } \\
\text { alimentar }\end{array}$ & 7 & 14,3 & 10 & 20,4 & 7 & 14,3 & 22 & 44,0 & 3 & 6,1 \\
\hline $\begin{array}{l}\text { Capacidade de } \\
\text { trabalhar }\end{array}$ & 12 & 24,5 & 10 & 20,4 & 4 & 8,2 & 21 & 42,9 & 2 & 4,1 \\
\hline Capacidade de viajar & 16 & 32,7 & 11 & 22,4 & 5 & 10,2 & 16 & 32,7 & 1 & 2,0 \\
\hline $\begin{array}{l}\text { Depender de } \\
\text { médicos }\end{array}$ & 42 & 85,7 & 2 & 4,1 & 1 & 2,0 & 3 & 6,1 & 1 & 2,0 \\
\hline Estresse & 14 & 28,6 & 16 & 32,7 & 6 & 1,2 & 12 & 24,5 & 1 & 2,0 \\
\hline Vida sexual & 26 & 53,1 & 7 & 14,3 & 10 & 20,4 & 5 & 10,2 & 1 & 2,0 \\
\hline Aparência pessoal & 36 & 73,5 & 11 & 22,4 & 1 & 2,00 & 0 & 0 & 0 & 0 \\
\hline
\end{tabular}


$\mathrm{Na}$ Tabela 2 estão apresentadas as análises bivariadas. Observou-se que a percepção negativa de saúde foi maior entre mulheres $(71,4 \%)$, entre os pacientes com 50 anos ou mais $(51,7 \%)$ e entre aqueles que relataram que a doença renal interfere na vida diária, em comparação aos homens $(57,1 \%)$, entre os pacientes com até 50 anos (40,8\%) e entre aqueles que relataram que a doença não interfere na saúde $(77,4 \%)$.

Tabela 2 - Relação percepção e saúde com sexo, idade, interferência na vida e gasto de tempo com a doença

\begin{tabular}{|c|c|c|c|c|c|c|}
\hline & \multicolumn{6}{|c|}{ Percepção e saúde } \\
\hline & \multicolumn{2}{|c|}{ Positivo } & \multicolumn{2}{|c|}{ Negativo } & \multicolumn{2}{|c|}{ Total } \\
\hline & $\mathrm{n}$ & $f(\%)$ & $\mathbf{n}$ & $f(\%)$ & $\mathrm{n}$ & $\mathrm{f}(\%)$ \\
\hline \multicolumn{7}{|l|}{ Sexo } \\
\hline Masculino & 12 & 42,9 & 16 & 57,1 & 28 & 57,1 \\
\hline Feminino & 6 & 28,6 & 15 & 71,4 & 21 & 42,9 \\
\hline \multicolumn{7}{|l|}{ Idade } \\
\hline $20-29$ & 5 & 27,8 & 6 & 19,4 & 11 & 22,4 \\
\hline $30-39$ & 3 & 16,7 & 6 & 19,4 & 9 & 18,4 \\
\hline $40-49$ & 3 & 16,7 & 3 & 9,7 & 6 & 12,2 \\
\hline $50-59$ & 3 & 16,7 & 10 & 32,3 & 13 & 26,5 \\
\hline$>60$ & 4 & 22,2 & 6 & 19,4 & 10 & 20,4 \\
\hline \multicolumn{7}{|c|}{$\begin{array}{l}\text { Doença renal interfere } \\
\text { demais na vida }\end{array}$} \\
\hline Positivo & 12 & 66,7 & 5 & 27,8 & 18 & 36,7 \\
\hline Negativo & 25 & 80,6 & 6 & 19,4 & 31 & 63,3 \\
\hline Valor de $\mathrm{P}$ & \multicolumn{6}{|c|}{$<0,05$} \\
\hline \multicolumn{7}{|c|}{$\begin{array}{l}\text { Gasto de tempo } \\
\text { com a doença }\end{array}$} \\
\hline Verdadeiro & 9 & 50 & 7 & 38,9 & 18 & 36,7 \\
\hline Falso & 24 & 77,4 & 7 & 22,6 & 31 & 63,3 \\
\hline Valor de $\mathrm{P}$ & \multicolumn{6}{|c|}{$<0,042$} \\
\hline
\end{tabular}

Quando perguntado se a doença renal interfere na sua vida, observou-se que as pessoas mais jovens responderam que não existe interferência (54,6\%). O contrário aconteceu com os das faixas etárias mais elevadas (24), em que quase a metade $(48,6 \%)$ respondeu que há interferência da doença em suas vidas. Já quando questionados se o esforço físico interfere na sua qualidade de vida, este estudo revelou que 70,3\% afirmam que sim, conforme Tabela 3. 
Tabela 3 - Relação interferência na vida com esforço físico e idade

\begin{tabular}{|c|c|c|c|c|c|c|}
\hline & \multicolumn{6}{|c|}{ Doença renal interfere demais na vida } \\
\hline & \multicolumn{2}{|c|}{ Positivo } & \multicolumn{2}{|c|}{ Negativo } & \multicolumn{2}{|c|}{ Total } \\
\hline & $\mathrm{n}$ & $f(\%)$ & n & $f(\%)$ & n & $f(\%)$ \\
\hline \multicolumn{7}{|c|}{ Esforço físico } \\
\hline $\operatorname{Sim}$ & 26 & 70,3 & 8 & 72,7 & 35 & 71,4 \\
\hline Não & 8 & 72,7 & 3 & 27,3 & 14 & 28,6 \\
\hline Valor de $\mathrm{P}$ & \multicolumn{6}{|c|}{$<0,046$} \\
\hline \multicolumn{7}{|l|}{ Idade } \\
\hline $20-29$ & 6 & 16,2 & 5 & 45,5 & 11 & 22,4 \\
\hline $30-39$ & 8 & 21,6 & 1 & 9,1 & 9 & 18,4 \\
\hline $40-49$ & 5 & 13,5 & 1 & 9,1 & 6 & 12,2 \\
\hline $50-59$ & 13 & 35,1 & 0 & 0 & 13 & 26,5 \\
\hline$>60$ & 5 & 13,5 & 4 & 36,4 & 10 & 20,4 \\
\hline Valor de P & \multicolumn{6}{|c|}{$<0,046$} \\
\hline
\end{tabular}

\section{Discussão}

Neste estudo constatou-se uma percepção negativa da QV nas pessoas portadoras de Doença Renal Crônica, que apresentam menores escores nos domínios esforços físicos e cãibras, corroborando o estudo de Martins (7), que mostra as atividades cotidianas, o trabalho e as atividades domésticas comprometidas.

Partindo dessas ideias, notou-se que os pacientes em tratamento hemodialítico apresentam alterações que afetam os múltiplos sistemas corporais, e entre eles os que se destacam são o sistema cardiovascular e o músculo-esquelético (25), ficando caracterizado que a maioria dos pacientes não consegue desenvolver as atividades laborais (26).

As manifestações musculares em pacientes submetidos à hemodiálise são bastante comuns, especialmente naqueles em tratamento dialítico de longa duração. Alterações importantes, como cãibras musculares (27), fraqueza muscular (28), pele seca, dormência, estresse, entre outros, corroborando ao estudo de Vieira et al. (29), trazem repercussões, como mostra o estudo de Vidal, Sales e Escobar (30), em que mais de 70\% dos pacientes em tratamento dialítico declaram que sua saúde física interfere no desempenho de suas atividades de vida diária. Na presente pesquisa foi observado que em algumas respostas há proporções semelhantes em categorias opostas (exemplo: 32,7\% relataram que a coceira não incomoda, enquanto $30,6 \%$ afirmaram sentir um incômodo extremo). Isso se deve à grande variabilidade daidade e também por se tratar de um estudo transversalnão se pode afirmar se o problema da coceira (a exemplo) se dá pela condição crônica ou por outro problema associado. Contudo, o estudo de Alonso (31) considera o prurido uma das maiores manifestações cutâneas.

Todas essas alterações levam o paciente com IRC a ter um estilo de vida sedentário, como mostra o estudo de Trentini (28), que indica que a maioria apresentou dificuldades em fazer exercícios físicos. Numerosos estudos têm demonstrado que o exercício físico nesta população, aplicado durante o tratamento hemodialítico, têm efeitos benéficos (32), como a melhora da capacidade funcional e da hipertensão arterial (33). 
As dores causadas em $64,4 \%$ das pessoas aparecem durante a sessão de hemodiálise, e manifestamse como contraturas musculares (cãibras), especialmente em extremidades inferiores (34), superiores e dorso, seguidos de dor abdominal e cefaleia (30).

Não houve diferença no nível de QV, no que se refere ao sexo. Porém, houve correlação linear negativa entre idade e as seguintes dimensões de QV: capacidade funcional (35), limitações por aspectos físicos e estado geral de saúde, como mostra o estudo de Santos (36).

Na categoria de função física alcançou uma média de 64,7\%, e em análise de seus itens se detectou que $76,7 \%$ dos pacientes apresentaram algum grau de limitação. Isso se deve não só à insuficiência renal, como também às complicações que acarretam esta patologia. Portanto, as atividades físicas que esses pacientes podem realizar devem ser aconselhadas de forma individual (37), levando em conta fatores como suas situações clínicas, idade, tipo de tratamento (30).

Corroborando o estudo de Martins (7), foi visto que atividades que requerem algum tipo de esforço evidenciam problemas na saúde física em 70,3\%, indicando prejuízo na QV.

Avaliando a categoria sexo e concordando com o estudo de Lopes (38), viu-se que 71,4\% das mulheres apresentam níveis mais baixos de QV que os homens, quando comparados aspectos de visão negativa de sua saúde.

Assim como no estudo de Welter (39), o prurido também é um sintoma marcante em pacientes em unidade de hemodiálise, em que a relação entre prurido e QV foi observada.

Dados encontrados neste estudo indicam que pacientes com IRC podem apresentar diminuição do desempenho funcional e prejuízo na prática de atividade física. Portanto, esses achados contribuem para melhor opção terapêutica, otimizando o tratamento clínico e auxiliando profissionais em suas intervenções, como evidenciado no trabalho de Coelho (40).

O recente estudo de Segura-Ortí (12) aponta a importância da fisioterapia para uma equipe multidisciplinar de saúde atuando no tratamento e avaliação da saúde comparada à QV de pessoas com doença renal crônica e suas atividades cotidianas, que são comprometidas com o tempo, promovendo transformações condizentes com a realidade deste estudo, podendo prevenir o comprometimento das atividades de vida diária, dados que também são evidenciados em outro trabalho (7).

\section{Considerações finais}

Os dados encontrados neste estudo indicam que pacientes com IRC apresentam diminuição na qualidade de vida por causa das repercussões musculo-esqueléticas, tais como cãibras, fraqueza muscular e esforço físico.

Após realizada a revisão bibliográfica para este estudo, foi visto que a atividade física apresenta-se de forma benéfica quanto à capacidade funcional e QV desses pacientes. Este estudo oferece subsídios para implementação de novas estratégias para melhoria da QV, uma vez que o indivíduo tem sua estrutura modificada e, com isso, mexe com suas emoções, com seus sentidos e com sua autoestima, afastando-se cada vez mais do seu meio social pela imagem que ele faz de si, tornando-o diferente das outras pessoas, gerando sentimento de angústia e tentativas de isolamento. Portanto, a fisioterapia pode auxiliar elaborando um programa de exercícios, com intensidade, frequência e duração, devendo estas serem adequadas às realidades de cada serviço e de cada paciente, respeitando seus limites, idade e capacidade física.

\section{Referências}

1. Moreno AB, Faerstein E, Werneck GL, Lopes CS, Chor D. Propriedades psicométricas do instrumento abreviado de avaliação de qualidade de vida da Organização Mundial da Saúde no Estudo Pró-Saúde. Cad Saúde Pública. 2006;22(12):2585-97. 
2. Pagani TC, Pagani Junior CR. Instrumentos de avaliação de qualidade de vida relacionada à saúde. Fundação Nacional de Desenvolvimento do Ensino Superior Particular. [Acesso em 18 de Out. 2008]. Disponível em: http:// www.faculdadecomunitaria.edu.br/programasinst/Revistas/revistas2006/rev_ciencias_bio/05.pdf

3. Silva DK, Nahas MV. Atividade física habitual e qualidade de vida relacionada à saúde em mulheres com doença vascular periférica. Rev Bras Ci Mov. 2004;12(4):63-8.

4. Kusumoto L, Marques S, Haas VJ, Rodrigues RA. Adults and elderly on hemodialysis evaluation of health related quality of life. Acta Paul Enferm. 2008;21(Número especial):152-59.

5. Dyniewicz AM, Zanella E, Kobus. Narrativa de uma cliente com insuficiência renal crônica: a história oral como estratégia de pesquisa. Rev Eletrôn Enfer. 2004;6(2):199-212.

6. Sarnak MJ, Levey AS, Schoolwerth AC, Coresh J, Culleton B, Hamm LL, et al. Kidney Disease as a risk factor for development of cardiovascular disease: a statement from the American Heart Association Councils on Kidney in Cardiovascular Disease, High Blood Pressure Research, Clinical Cardiology, and Epidemiology and Prevention. Hypertension of the American Heart Association. Hypertension. 2003;42(5):1050-65.

7. Martins MR, Cesarino CB. Qualidade de vida de pessoas com doença renal crônica em tratamento hemodialítico. Rev Latino-Am Enfer. 2005;13(5):670-6.

8. Barros E, Gonçalves LF. Nefrologia no consultório. São Paulo: Artmed; 2007.

9. Queiroz MVO, Dantas MCQ, Ramos IC, Jorge MSB. Tecnologia do cuidado ao paciente Renal Crônico: enfoque educativo-terapêutico a partir das necessidades dos sujeitos. Texto \& Contexto Enferm. 2008;17(1):55-63.

10. Ramos IC, Queiroz MVO, Jorge MSB. Cuidado em situação de Doença Renal Crônica: representações sociais elaboradas por adolescentes. Rev Bras Enferm. 2008;61(2):193-200.

11. Brasil. Ministério da Saúde. Secretaria de Políticas de Saúde. Departamento de Atenção Básica. Prevenção clínica de doenças cardiovasculares e renais. Ministério da Saúde, Secretaria de Atenção à Saúde, Departamento de Atenção Básica. Brasília: Ministério da saúde, 2006. (Cadernos de Atenção Básica, 14).

12. Leite IC, Schramm JM, Gadelha AM, Valente JG, Campos MR, Portela MC, et al. Comparação das informações sobre as prevalências de doenças crônicas obtidas pelo suplemento saúde da PNAD/98 e as estimadas pelo estudo Carga de Doença no Brasil. Ciência \& Saúde Coletiva. 2002;7(4):733-41.

13. Santos $\mathrm{AB}$, Bandeira MA, Coiado CRP. Avaliação do grau de depressão em pacientes com insuficiência renal crônica submetidos à hemodiálise. Nursing (São Paulo). 2008;11(124):411-8.

14. Higa K, Kost MT, Soares DM, Morais MC, Polins BRG. Qualidade de vida de pacientes portadores de insuficiência renal crônica em tratamento de hemodiálise. Acta Paul Enferm. 2008;21(número especial):203-6.

15. Almeida AM. Revisão: a importância da saúde mental na qualidade de vida e sobrevida do portador de insuficiência renal crônica. J Bras Nefrol. 2003;25(4):209-14.

16. Segura-Ortí E, Rodilla-Alama V, Lisón JF. Fisioterapia durante la hemodiálisis: resultados de um programa de fuerza-resistencia. Nefrologia. 2008;28(1)67-72.

17. Chaves LD, Anselmi ML, Barbeira CB, Hayashida M. Estudo da sobrevida de pacientes submetidos a hemodiálise e estimativa de gastos no município de Ribeirão Preto-SP. Rev Esc Enferm. 2002;36(2):193-9.

18. Sociedade Brasileira de Nefrologia. Censo de Diálise SNB 2008: pacientes em diálise no Brasil, por Região, mar. 2008. [acesso em 8 out. 2008]. Disponível em: http://www.sbn.org.br/censos.htm

19. Kusumota L. Avaliação da qualidade de vida relacionada à saúde de pacientes em hemodiálise [tese]. Ribeirão Preto: Escola de Enfermagem de Ribeirão Preto, Universidade de São Paulo; 2005. 
20. Terra FS, Costa AM. Avaliação da qualidade de vida de pacientes renais crônicos submetidos à hemodiálise. Rev enferm UERJ. 2007;15(3):430-6.

21. Duarte PS, Miyazaki MC, Ciconelli RM, Sesso R. Tradução e adaptação cultural do instrumento de avaliação de qualidade de vida para pacientes renais crônicos (KDQOL-SF TM). Rev Assoc Med Bras. 2003;49(4): 375-81.

22. Duarte PS, Ciconelli RM, Sesso R. Cultural adaptation and validation of the "Kidney Disease and Quality of Life Short Form (KDQOL-SFTM 1.3)” in Brazil. Braz J Med Biol Res. 2005;38(2):261-70.

23. Pacheco GS, Santos I, Bregman R. Características de clientes com doença renal crônica: evidências para o ensino do autocuidado. Rec Enferm UERJ. 2006;14(3):434-9.

24. Souza FF. Avaliação da qualidade de vida do idoso em hemodiálise: comparação de dois instrumentos genéricos [dissertação]. Campinas: Universidade Estadual de Campinas; 2004.

25. Reboredo MM, Henrique DMN, Bastos MG, Paula RB. Exercício físico em pacientes dialisados. Rev Bras Med Esporte. 2007;13(6):427-30.

26. Lara EA, Sarquis LM. O paciente renal crônico e sua relação com o trabalho. Cogitare Enferm. 2004;9(2): 99-106.

27. Nascimento CD, Marques IR. Intervenções de enfermagem nas complicações mais freqüentes durante a sessão de hemodiálise: revisão da literatura. Rev Bras Enferm. 2005;58(6):719-22.

28. Trentini M, Corradi EM, Araldi MA, Tigrinho FC. Qualidade de vida de pessoas dependentes de hemodiálise considerando alguns aspectos físicos, sociais e emocionais. Texto \& Contexto Enferm. 2004;13(1): 74-82.

29. Vieira WP, Gomes KW, Frota NB, Andrade JE, Vieira RM, Moura FE, et al. Manifestações musculoesqueléticas em pacientes submetidos à hemodiálise. Rev Bras Reumatol. 2005;6(45):357-64.

30. Vidal MR, Salas MC, Escobar JM. Calidad de vida em pacientes renales hemodializados. Cienc Enferm. 2005;11(2):47-55.

31. Alonso C, Del Coro V, Di Nicolantonio MA, Porfiri G, Nicola G, Acosta F, et al. Manifestaciones cutáneas en pacientes hemodializados: nuestra experiência. Arch Argent Dermatol. 2006;56(1):17-22.

32. Segura-Ortí E, Momblanch T, Martínez JF, Martí-i-Monrós A, Tormo G, Lisón-Párraga JF. Programa de ejercicio para pacientes con insuficiencia renal crónica en hemodiálisis: estudio piloto. Rev Soc Esp Enferm Nefrol. 2007;10(3):84-6.

33. Henrique DMN, Paula RB. Exercício físico na doença renal crônica [dissertação]. Juiz de Fora: Faculdade de Medicina da Universidade Federal de Juiz de Fora; 2009.

34. Lins SMB, Linhares FMP. Proposta de protocolo para consulta de enfermagem a pacientes portadores de insuficiência renal crônica em tratamento conservador. Enfermagem Brasil. 2007;6(5):305-11.

35. Castro M, Caiuby AVS, Draibe AS, Canziani MEF. Qualidade de vida de pacientes com insuficiência renal crônica em hemodiálise avaliada através do instrumento genérico SF-36. Rev Assoc Med Bras. 2003;49(3): 245-9.

36. Santos PR. Relação do sexo e da idade com nível de qualidade de vida em renais crônicos hemodialisados. Rev Assoc Med Bras. 2006;52(5):356-9.

37. Moura RMF, Silvia SCR, Ribeiro GM, Sousa LA. Efeito do exercício físico durante a hemodiálise em indivíduos com insuficiência renal crônica: uma revisão. Fisioter Pesq. 2008;15(1):86-91. 
38. Lopes GB, Martins MT, Matos CM, Amorim JL, Leite EB, Miranda EA, et al. Comparações de medidas de qualidade de vida entre mulheres e homens em hemodiálise. Rev Assoc Med Bras. 2007;53(6):506-9.

39. Welter EQ, Bonfá R, Petry V, Moreira LL, Weber MB. Relação entre grau de prurido e qualidade de vida de pacientes em hemodiálise. An Bras Dermatol. 2008;83(2):137-40.

40. Coelho CC, Aquino ES, Lara KL, Peres TM, Barja PR, Lima EM. Repercussões da insuficiência renal crônica na capacidade de exercício, estado nutricional, função pulmonar e musculatura respiratória de crianças e adolescentes. Rev Bras Fisioter. 2008;12(1):1-6.

Recebido: $17 / 12 / 2008$

Received: $12 / 17 / 2008$

Aprovado: 18/03/2010 Approved: 03/18/2010

Revisado: 24/06/2010

Reviewed: 06/24/2010 